\title{
Thermodynamic Quantities of Imbalanced 2D Fermi Gases Near the Berezinskii-Kosterlitz-Thouless Transition
}

\author{
J. Tempere • S.N. Klimin • J.T. Devreese • \\ B. Van Schaeybroeck
}

Received: 13 June 2009 / Accepted: 17 August 2009

(C) Springer Science+Business Media, LLC 2009

\begin{abstract}
We investigate the effects of imbalance on the two-dimensional BerezinskiiKosterlitz-Thouless superfluid transition for a Fermi gas in a parabolic trap. Thermodynamic parameters of the Fermi gas are determined using the functional integral formalism in combination with a hydrodynamic action functional for the phase fluctuations.
\end{abstract}

Keywords Quantum gases · Imbalance · BKT transition · Low dimensions

PACS 03.75.Ss $\cdot$ 05.30.Fk $\cdot$ 03.75.Lm

\section{Introduction}

Recent progress in the experimental investigation of ultra-cold atoms stimulated an unprecedented interest in the theoretical problems of condensation of cold bosons and pairing of interacting fermions (see, e.g., the review [1] and references therein). The interaction strength between atoms can be tuned using Feshbach resonances, and

S.N. Klimin on leave from Dept. of Theoretical Physics, State Univ. of Moldova, MD-2009 Chisinau, Moldova.

J.T. Devreese also at COBRA, Eindhoven University of Technology, 5600 MB, Eindhoven, The Netherlands.

J. Tempere $(\bowtie) \cdot$ S.N. Klimin · J.T. Devreese

TFVS, Universiteit Antwerpen, 2020 Antwerpen, Belgium

e-mail: jacques.tempere@ua.ac.be

J. Tempere

Lyman Laboratory of Physics, Harvard University, Cambridge, MA 02138, USA

B. Van Schaeybroeck

Institute of Theoretical Physics, KULeuven, Celestijnenlaan 200D, 3001 Heverlee, Belgium 
this allows to observe an evolution of a system of atoms from a Bardeen-CooperSchrieffer (BCS) type of pairing to a Bose-Einstein condensate (BEC) of molecules. One of the striking experimental results on ultracold fermion atoms is the observation of phase separation of a two-component system in the case of an imbalance of populations of "spin up" and "spin down" fermions [2-5]. Here "spin-up" and "spin-down" refer to the two states which pair up to form Cooper pairs or molecules.

Advances in pairing of trapped cold atomic Fermi gases with a controlled geometry of a trapping potential allow experimentalists to realize systems of different (quasi-) dimensionalities. Both condensation of bosonic atoms [6] and pairing of fermions [7] have been observed recently in optical lattices. The Mermin-WagnerHohenberg theorem [8,9] shows that in a uniform, two-dimensional (2D) system, the long range order is destroyed by thermal fluctuations so that $2 \mathrm{D}$ Bose gases cannot undergo Bose-Einstein condensation at nonzero temperatures [10-12]. The same feature is manifested in the interacting Fermi gas in 2D as shown by Schmitt-Rink, Varma and Ruckenstein [13]. Nevertheless, a superfluid state in boson and fermion two-dimensional systems can be stabilized through the BerezinskiiKosterlitz-Thouless (BKT) mechanism [14-16]. The BKT mechanism plays a role in the stabilization of superfluidity also in trapped systems [17]. A BKT-type crossover in a trapped quantum degenerate gas of ${ }^{87} \mathrm{Rb}$ atoms has recently been observed by Hadzibabic et al. [18]. This has stimulated renewed theoretical interest in the BKT transition in trapped dilute gases [19-24].

Strictly speaking, the Mermin-Wagner-Hohenberg theorem is valid only in uniform $2 \mathrm{D}$ systems. The trapped $2 \mathrm{D}$ and quasi $2 \mathrm{D}$ gases can pass a true $\mathrm{BEC}$ phase transition, as well as the BKT phase transition. As shown by Holtzmann et al. [25, 26], the BKT phase transition for quasi 2D systems can be realized in strongly anisotropic traps.

The BKT phase transition for a balanced 2D Fermi gas with the $s$-wave scattering in the balanced case has been theoretically analyzed in Refs. [27, 28]. They treated the fermion pairing in $2 \mathrm{D}$ by the path integral technique taking into account phase fluctuations. In Ref. [29], the approach of Ref. [27] has been extended to investigate the effect of population imbalance on the BKT phase transition in a 2D Fermi gas. On the basis of a hydrodynamic effective bosonic action, in Ref. [29] phase diagrams for an interacting, imbalanced Fermi gas in 2D have been analyzed.

In Refs. [30, 31], spectra of collective excitations for a 2D and 3D fermion gas in a harmonic trap were calculated suggesting the BCS phase transition at the boundary of the superfluid core. The thermodynamic parameters of the fermion gas were determined in the low-temperature approximation within the mean-field approach at $T=0$. The investigation of collective excitations of imbalanced fermion gases in Refs. $[30,31]$ is performed using linearized Euler and continuity equations in the superfluid phase and the Boltzmann-Vlasov equation in the normal phase. Here, using the approach of Ref. [29], we investigate thermodynamic quantities relevant for the superfluid hydrodynamics of a trapped Fermi gas. 


\section{Path-Integral Approach}

A two-component gas of interacting fermions in 2D with the $s$-wave pairing and with a population imbalance is treated within the path-integral formalism. The partition function of the system of fermions in 2D is expressed as the path integral over Grassmann variables $\left[\bar{\psi}_{\mathbf{x}, \tau, \sigma}, \psi_{\mathbf{x}, \tau, \sigma}\right]$,

$$
\mathcal{Z} \propto \int \mathcal{D}\left[\bar{\psi}_{\mathbf{x}, \sigma}(\tau), \psi_{\mathbf{x}, \sigma}(\tau)\right] \exp (-S)
$$

The action functional of interacting fermions with the $s$-wave pairing is a sum of the free-fermion and interaction terms,

$$
\begin{aligned}
S= & \int_{0}^{\beta} d \tau \int d^{2} \mathbf{x} \sum_{\sigma=\uparrow, \downarrow}\left[\bar{\psi}_{\mathbf{x}, \sigma}(\tau)\left(\frac{\partial}{\partial \tau}-\nabla_{\mathbf{x}}^{2}-\mu_{\sigma}\right) \psi_{\mathbf{x}, \sigma}(\tau)\right] \\
& +g \int_{0}^{\beta} d \tau \int d^{2} \mathbf{x} \int d^{2} \mathbf{y} \bar{\psi}_{\mathbf{x}, \uparrow}(\tau) \bar{\psi}_{\mathbf{y}, \downarrow}(\tau) \psi_{\mathbf{y}, \downarrow}(\tau) \psi_{\mathbf{x}, \uparrow}(\tau),
\end{aligned}
$$

where $\beta=\frac{1}{k_{B} T}$ is the inverse to the temperature. The contact interaction in (2) gives rise to ultraviolet divergences. They are removed by the renormalization procedure as in Refs. $[32,33]$. The strength $g$ of the contact interaction is expressed through the two-particle binding energy $E_{b}$ in $2 \mathrm{D}$ by the equation [33]:

$$
\frac{1}{g}=\frac{1}{8}\left[i-\frac{1}{\pi} \ln \left(\frac{E}{E_{b}}\right)\right]-\int \frac{d^{2} \mathbf{k}}{(2 \pi)^{2}} \frac{1}{2 k^{2}-E+i \delta} \quad(\delta \rightarrow+0) .
$$

Here, we use the units in which $\hbar=1$, the fermion mass $m=1 / 2$, and the Fermi energy $E_{F} \equiv\left(2 \pi n_{0}\right)^{2 / 3} /(2 m)=1$ (where $n_{0}$ is the fermion density in $2 \mathrm{D}$ ). The results below are expressed in terms of the averaged chemical potential $\mu=\left(\mu_{\uparrow}+\mu_{\downarrow}\right) / 2$ determining the total number of fermions and the chemical potential imbalance $\zeta=\left(\mu_{\uparrow}-\mu_{\downarrow}\right) / 2$. Further on, we apply the Hubbard-Stratonovich (HS) transformation with the auxiliary Bose field (HS field) $\phi_{\mathbf{r}}(\tau)$. After integrating out the fermion Grassmann variables, an effective action in the bose field $\phi_{\mathbf{r}}$ is obtained. The remaining functional integral over this Bose field cannot be taken in general. Several levels of approximation can be made to get results. The crudest approximation is the meanfield approximation which replaces the field by a constant, $\phi_{\mathbf{r}} \rightarrow \Delta$, the saddle point. To improve on this, fluctuations around the saddle point can still be taken into account; this can be done in an exact way only up to quadratic order in the fluctuation. In order to treat the quasicondensate state, relevant for the $2 \mathrm{D}$ case, we have to focus on phase fluctuations: that corresponds to setting $\phi_{\mathbf{r}} \rightarrow \Delta e^{i \theta_{\mathbf{r}}}$. We assume that the remaining fluctuation field $\theta_{\mathbf{r}}$ varies slowly as a function of position and time with respect to the variations of the fermion fields. A similar assumption was used in Refs. $[27,28,34]$. The resulting hydrodynamic action, which is obtained after the quadratic expansion in powers of phase fluctuations is the sum of the saddle-point action $S_{s p}$, which coincides with the expression (5) of Ref. [33], and the diagonal quadratic form 
of the phase fluctuations

$$
S_{f l}=\frac{1}{2} \int_{0}^{\beta} d \tau \int d^{2} \mathbf{r}\left[A\left(\frac{\partial \theta}{\partial \tau}\right)^{2}+\rho_{s}(\nabla \theta)^{2}\right] .
$$

The coefficients in the fluctuation action are the pair superfluid density

$$
\rho_{s}=\int \frac{d^{2} \mathbf{k}}{(2 \pi)^{2}}\left[\frac{1}{2}\left(1-\frac{\xi_{k}}{E_{k}} \frac{\sinh \beta E_{k}}{\cosh \beta \zeta+\cosh \beta E_{k}}\right)-\frac{k^{2}}{2} \beta \frac{\cosh \beta \zeta \cosh \beta E_{k}+1}{\left(\cosh \beta \zeta+\cosh \beta E_{k}\right)^{2}}\right]
$$

and the constant

$$
A=\frac{1}{4} \int \frac{d^{2} \mathbf{k}}{(2 \pi)^{2}}\left(\frac{\Delta^{2}}{E_{k}^{3}} \frac{\sinh \beta E_{k}}{\cosh \beta \zeta+\cosh \beta E_{k}}+\frac{\xi_{k}^{2}}{E_{k}^{2}} \beta \frac{\cosh \beta \zeta \cosh \beta E_{k}+1}{\left(\cosh \beta \zeta+\cosh \beta E_{k}\right)^{2}}\right),
$$

where $\xi_{k}=k^{2}-\mu$ is the fermion energy, and $E_{k}=\sqrt{\xi_{k}^{2}+\Delta^{2}}$ is the Bogolubov excitation energy.

The action in (4) describes a Bose gas of spin waves [27] with the energy spectrum $\omega_{k}=c k$, where $c=\sqrt{\rho_{s} / A}$ is the sound velocity. The spin wave contribution to the thermodynamic potential per unit area is given by the expression $\Omega_{s w}=\frac{1}{\beta} \int \frac{d^{2} \mathbf{k}}{(2 \pi)^{2}} \ln \left(1-e^{-\beta \omega_{k}}\right)$. The total thermodynamic potential of the fermion system taking into account phase fluctuations is the sum of the above spin-wave term and the saddle-point contribution, which is provided by the saddle-point action, $\Omega_{s p}=\int \frac{d^{2} \mathbf{k}}{(2 \pi)^{2}}\left[\frac{1}{\beta} \ln \left(2 \cosh \beta \zeta+2 \cosh \beta E_{k}\right)-\xi_{k}\right]-\frac{\beta}{g} \Delta^{2}$. For an imbalanced quasi 2D Fermi gas in an optical potential, the mean-field zero-temperature phase diagrams were analyzed in Ref. [33] on the basis of this saddle-point action (neglecting spin-wave contributions).

The gap parameter $\Delta$ for an imbalanced Fermi gas is determined through the minimization of the saddle-point thermodynamic potential $\Omega_{s p}$ as a function of $\Delta$ at given $\beta, \mu, \zeta$. For a complete determination of thermodynamic parameters at a given temperature, the minimum condition for the thermodynamic potential is solved jointly with the number equations. The BKT mechanism of the superfluid phase transition in $2 \mathrm{D}$ boson and fermion systems consists in the formation of bound vortex-antivortex pairs below a critical temperature $T_{B K T}$. Above $T_{B K T}$, free vortices and antivortices proliferate spoiling the phase coherence. The BKT transition temperature is obtained as a joint solution of the equation [35] $T_{B K T}-\frac{\pi}{2} \rho_{S}\left(T_{B K T}\right)=0$ together with the coupled gap and number equations as described in Ref. [29].

\section{Discussion and Results}

In Fig. 1(a), we plot the pressure of the imbalanced fermion gas in 2D, as determined from the thermodynamic potential $\Omega_{s p}+\Omega_{s w}$, as a function of the temperature for different values of the chemical potential imbalance at $E_{b}=0.1$, where $E_{b}$ is the binding energy of a two-particle bound state in 2D. The energy $E_{b}$ characterizes the coupling strength of the fermion-fermion attractive interaction. 

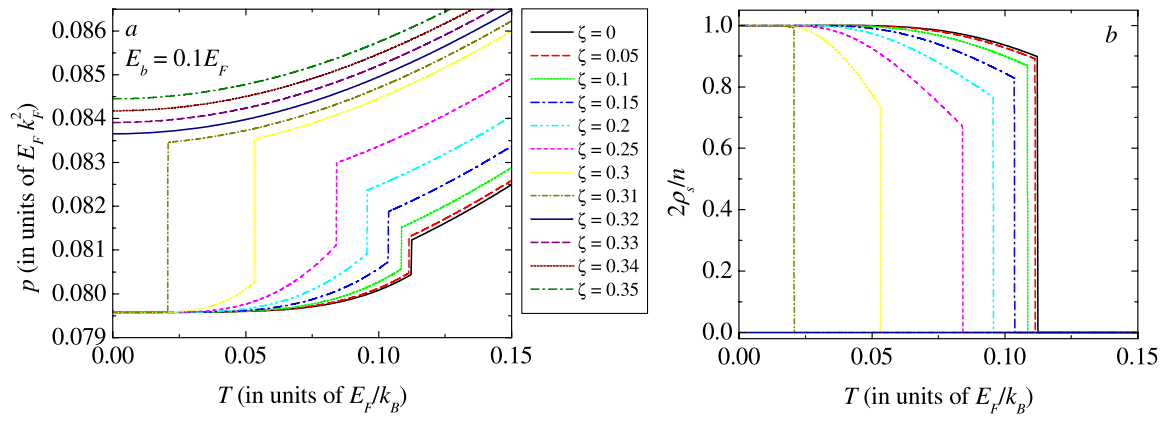

Fig. 1 (Color online) Pressure (a) and superfluid density (b) of an imbalanced fermion gas in 2D as a function of the temperature at the binding energy $E_{b}=0.1 E_{F}$ for different values of the chemical potential imbalance

For sufficiently small values of $\zeta$, including $\zeta=0$, at low temperatures the pressure slowly increases with increasing $T$, and reveals a jump at the BKT transition temperature $T=T_{B K T}$. The BKT transition temperature falls down with increasing $\zeta$. Starting from a certain critical value $\zeta \equiv \zeta_{0}$ (which lies in the range $0.31<\zeta_{0}<0.32$ for $E_{b}=0.1$ ), there is no jump for the pressure. This means that the BKT transition at finite temperatures does not occur for $\zeta>\zeta_{0}$. This conclusion is confirmed by the results for the superfluid density $\rho_{s}$ plotted in Fig. 1(b). We can see that for sufficiently high chemical potential imbalance, $\rho_{s}$ is equal to zero for all considered temperatures. For sufficiently low values of $\zeta$ (such that a finite $T_{B K T}$ exists), the superfluid density slowly lowers with increasing temperature below $T_{B K T}$.

In the low-temperature case, the pressure practically does not depend on $\zeta$ for $\zeta<\zeta_{0}$. This result is explained by the fact that at $T=0$, the relative population imbalance $\frac{\delta n}{n}=0$ for $\zeta<\zeta_{0}$. For $\zeta>\zeta_{0}$, the pressure increases with increasing the chemical potential imbalance at all temperatures.

In order to study fermions in a trap, we apply the local density approximation, in which the density $n$ and the density difference $\delta n$ depend on coordinates through the coordinate-dependent chemical potential $\mu(r)$. The normalization conditions are

$$
2 \pi \int_{0}^{\infty} n(\mu(r)) r d r=N, \quad 2 \pi \int_{0}^{\infty} \delta n(\mu(r)) r d r=\delta N .
$$

For a state in a parabolic trap without a vortex, the coordinate-dependent local chemical potential is $\mu(r)=\mu_{0}-\omega^{2} r^{2} / 4$, where $\omega$ is a confinement frequency of a parabolic potential. The pressure and the chemical potential for a trapped imbalanced $2 \mathrm{D}$ Fermi gas are calculated as a function of the local density at finite temperatures taking into account phase fluctuations, which are necessary to provide the BKT superfluid phase transition.

As pointed out above, a true BCS-BEC superfluid phase transition for interacting fermions in $2 \mathrm{D}$ is not possible at a finite temperature. Instead, for interacting fermions in 2D the BKT phase transition occurs at a finite temperature. In this connection, the equilibrium size and shape of distributions for thermodynamic parameters (e.g., fermion density and pressure), in general, differ from those used in Ref. [30]. As a result, the spectrum of collective modes for a 2D Fermi gas with the BKT transition will 

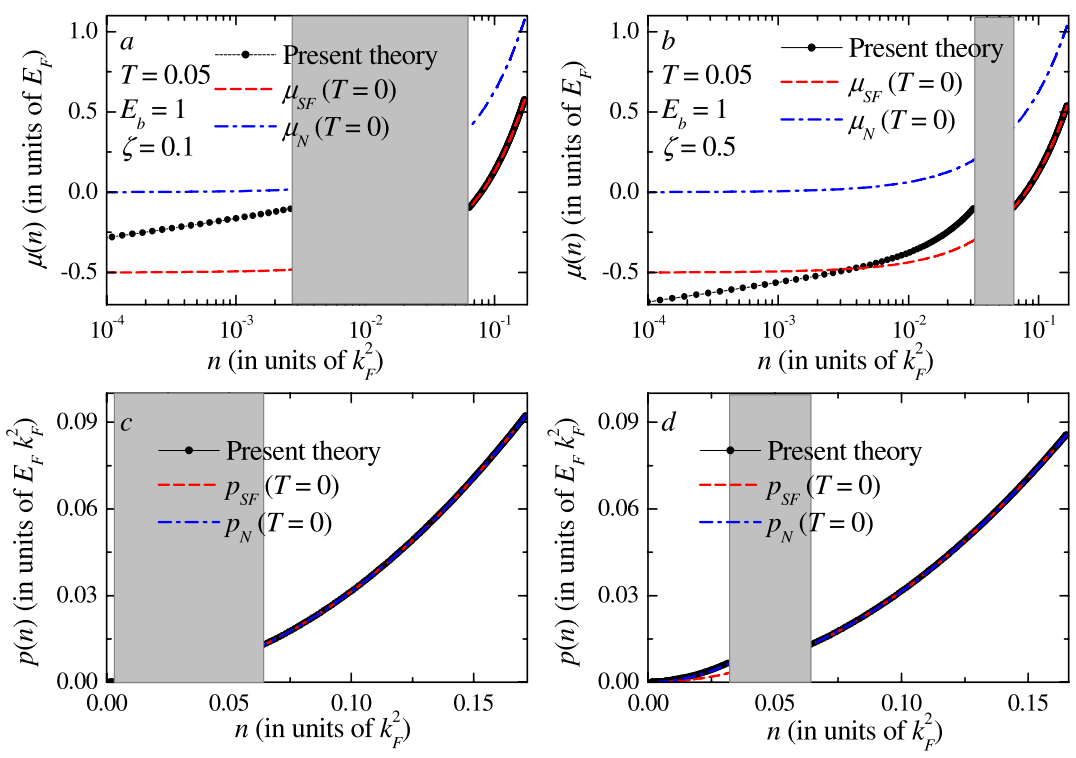

Fig. 2 (Color online) Chemical potential $(\mathbf{a}, \mathbf{b})$ and pressure $(\mathbf{c}, \mathbf{d})$ as a function of the local density for trapped fermions in 2D for $T=0.05, E_{b}=1$ and $\zeta=0.1(\mathbf{a}, \mathbf{c})$ and $0.5(\mathbf{b}, \mathbf{d})$. Full dots: the numerical finite-temperature calculation. Red dashed curves: analytic expressions for the superfluid phase at $T=0$. Blue dot-dashed curves: analytic expressions for the normal phase at $T=0$. Shaded areas indicate a jump of the local density $n$ at the phase boundary

differ from that for a gas with the BCS phase transition. Furthermore, at finite temperatures the dependence of thermodynamic parameters on the local density differs from analytic expressions for $T=0$ used in Ref. [30]. In general, those dependences can be obtained only numerically. In the present investigation, we supplement numerical results for the pressure and for thermodynamic potential with their fitting by analytic formulae in order to make possible to formulate equations for frequencies of collective modes in an analytic form similarly to Ref. [30].

For $N \gg 1$ fermions in a parabolic trap with the frequency parameter $\omega$, the Fermi energy is $E_{F} \approx \hbar \omega N^{1 / 2}$. The chemical potential is measured in units of $E_{F}$. The local density is measured in units of $k_{F}^{2}$, where $k_{F} \equiv \sqrt{E_{F}}$. Therefore also the density is measured in units of $E_{F}$. The pressure is measured in units of the energy per unit area, so that the unit for $p$ is $E_{F} k_{F}^{2}=E_{F}^{2}$. The local density varies keeping constant values of the chemical potential imbalance $\zeta \equiv\left(\mu_{\uparrow}-\mu_{\downarrow}\right) / 2$, what is relevant for fermions in a trap. The gap parameter for each value of the density is determined self-consistently from the minimum condition for the saddle-point thermodynamic potential.

In Fig. 2, the chemical potential (panels " $a, b$ ") and the pressure (panels " $c, d$ ") are plotted as a function of the local density $n$ for $T=0.05$ and $E_{b}=1$ with $\zeta=0.1$ and 0.5 . Full dots indicate results of the numerical calculation within the present theory. Dashed red curves show analytic expressions for the averaged chemical potential $\mu \equiv$ $\left(\mu_{\uparrow}+\mu_{\downarrow}\right) / 2$ and for the pressure $p$ in the superfluid phase at $T=0$ corresponding to Ref. [30]: $\mu_{S F}=2 \pi n-\frac{E_{b}}{2}$ and $p_{S F}=\pi n^{2}$, where $E_{b}$ is the binding energy of a 
two-particle bound state in 2D. Dot-dashed blue curves represent analytic expressions after Ref. [30] for the chemical potential and for the pressure in the normal phase at $T=0: \mu_{N}=2 \pi n$ and $p_{N}=\pi\left(n^{2}+\delta n^{2}\right)$.

As shown in Ref. [29], phase diagrams of $2 \mathrm{D}$ fermion systems with a population imbalance can contain the phase-separated state and the tricritical point similarly to phase diagrams of 3D fermion systems $[36,37]$. The local density of imbalanced trapped fermions in 3D has a jump at the bound of the superfluid core. Similarly, the density of imbalanced trapped fermions in 2D at sufficiently high binding energies (what is the case in Fig. 2, where $E_{b}=1$ ) can behave discontinuously at the phase boundary owing to imbalance.

The numerically calculated pressure slightly differs from the analytic formulae for $p_{S F}$ and $p_{N}$ given above. Also the numerically calculated chemical potential in the superfluid phase is close to $\mu_{S F}$ (but not exactly the same). However, the chemical potential in the normal phase provided by our theory substantially differs from $\mu_{N}$.

\section{Conclusions}

We have described the effects of imbalance on the BKT superfluid transition in a 2D Fermi gas, through the functional integral formalism and using the hydrodynamic effective action for phase fluctuations. Due to the rise of the upper-temperature bound of the instability region, the critical temperature of the BKT phase transition at a fixed binding energy falls down with an increasing imbalance. Therefore a population imbalance is a factor destroying superfluidity in 2D systems. The BKT transition can be experimentally observable for an imbalanced Fermi gas through the phase separation in a quasi 2D trap. The parameters of the state of that system (critical temperatures, pressure, superfluid density) are very sensitive to the imbalance. In a parabolic trap, In the $2 \mathrm{D}$ imbalanced system, similarly to a fermion system in a $3 \mathrm{D}$ parabolic trap, a phase separation of normal and superfluid phases occurs at the phase boundary. Because of imbalance, the thermodynamic parameters exhibit a discontinuous behavior at the phase boundary. The numerically calculated dependence of the pressure on the local density in a trap shows relatively small fluctuation corrections with respect to the analytic expression from Ref. [30]; the fluctuation corrections are more important for the chemical potential.

Acknowledgements This work was supported by FWO-V projects G.0115.06, G.0356.06, G.0180.09N, G.0370.09N, the WOG WO.033.09N (Belgium), and INTAS Project no. 05-104-7656. J.T. gratefully acknowledges support of the Special Research Fund of the University of Antwerp, BOF NOI UA 2004.

\section{References}

1. I. Bloch, J. Dalibard, W. Zwerger, Rev. Mod. Phys. 80, 885 (2008)

2. M.W. Zwierlein, A. Schirotzek, C.H. Schunck, W. Ketterle, Science 311, 492 (2006)

3. M.W. Zwierlein, C.H. Schunck, A. Schirotzek, W. Ketterle, Nature (London) 442, 54 (2006)

4. G.B. Partridge, W. Li, R.I. Kamar, Y.-A. Liao, R.G. Hulet, Science 311, 503 (2006)

5. Y. Shin, C.H. Schunck, A. Schirotzek, W. Ketterle, Nature (London) 451, 689 (2008)

6. M. Greiner, M.O. Mandel, T. Esslinger, T. Hänsch, I. Bloch, Nature 415, 39 (2002) 
7. J.K. Chin, D.E. Miller, Y. Liu, C. Stan, W. Setiawan, C. Sanner, K. Xu, W. Ketterle, Nature (London) 443, 961 (2006)

8. N.D. Mermin, H. Wagner, Phys. Rev. Lett. 17, 1133 (1966)

9. P.C. Hohenberg, Phys. Rev. 158, 383 (1967)

10. N.N. Bogolubov, Lectures on Quantum Statistics, vol. 2 (Gordon and Breach, New York, 1970)

11. E.M. Lifshitz, L.P. Pitaevskii, Statistical Physics (Pergamon, Oxford, 1980). Pt. 2

12. V.N. Popov, Functional Integrals in Quantum Field Theory and Statistical Physics (Reidel, Dordrecht, 1983)

13. S. Schmitt-Rink, C.M. Varma, A.E. Ruckenstein, Phys. Rev. Lett. 63, 445 (1989)

14. V.L. Berezinskii, Sov. Phys. JETP 32, 493 (1971)

15. J.M. Kosterlitz, D.J. Thouless, J. Phys. C 6, 1181 (1973)

16. J.M. Kosterlitz, J. Phys. C 7, 1046 (1974)

17. N. Prokof'ev, B. Svistunov, Phys. Rev. A 66, 043608 (2002)

18. Z. Hadzibabic, P. Krüger, M. Cheneau, B. Battelier, J. Dalibard, Nature 441, 1118 (2006)

19. S. Sachdev, E. Demler, Phys. Rev. B 69, 144504 (2004)

20. P. Krüger, Z. Hadzibabic, J. Dalibard, Phys. Rev. Lett. 99, 040402 (2007)

21. M. Holzmann, G. Baym, J.-P. Blaizot, F. Laloë, Proc. Natl. Acad. Sci. USA 104, 1476 (2007)

22. Z. Hadzibabic, P. Krüger, M. Cheneau, S.P. Rath, J. Dalibard, New J. Phys. 10, 045006 (2008)

23. T.P. Simula, M.J. Davis, P.B. Blakie, Phys. Rev. A 77, 023618 (2008)

24. R.N. Bisset, M.J. Davis, T.P. Simula, P.B. Blakie, Preprint arXiv:0804.0286 (2008)

25. M. Holtzmann, W. Krauth, Phys. Rev. Lett. 100, 190402 (2008)

26. M. Holzmann, M. Chevallier, W. Krauth, Europhys. Lett. 82, 30001 (2008)

27. S.S. Botelho, C.A.R. Sá de Melo, Phys. Rev. Lett. 96, 040404 (2006)

28. W. Zhang, G.-D. Lin, L.-M. Duan, Phys. Rev. A 78, 043617 (2008)

29. J. Tempere, S.N. Klimin, J.T. Devreese, Phys. Rev. A 79, 053637 (2009)

30. B. Van Schaeybroeck, A. Lazarides, Phys. Rev. A 79, 033618 (2009)

31. B. Van Schaeybroeck, A. Lazarides, Phys. Rev. A 77, 041602 (2008)

32. M. Randeria, J.-M. Duan, L.-Y. Shieh, Phys. Rev. B 41, 327 (1990)

33. J. Tempere, M. Wouters, J.T. Devreese, Phys. Rev. B 75, 184526 (2007)

34. S. De Palo, C. Castellani, C. Di Castro, B.K. Chakraverty, Phys. Rev. B 60, 564 (1999)

35. D.R. Nelson, J.M. Kosterlitz, Phys. Rev. Lett. 39, 1201 (1977)

36. J. Tempere, S.N. Klimin, J.T. Devreese, V.V. Moshchalkov, Phys. Rev. B 77, 134502 (2008)

37. J. Tempere, S.N. Klimin, J.T. Devreese, Phys. Rev. A 78, 023626 (2008) 